\title{
Modeling Method of FLAC3D Based on RHINO-KUBRIX and Deformation Analysis of Tunnel Lining Structure
}

\author{
Mao Jiangnan ${ }^{1, a}$, Zuo Guorong ${ }^{2, b}$ \\ ${ }^{1}$ Chongqing Transport Commission, Chongqing 400000, China \\ ${ }^{2}$ Sinohydro Bureau 14 CO.LTD, Chongqing 405200, China \\ 346347821@qq.com a email, 857322510@qq.com ${ }^{\text {bemail }}$
}

\begin{abstract}
Keywords: FLAC3D numerical model, RHINO-KUBRIX, Tunnel Deformation, Ground Stress
Abstract. Numerical simulation is more and more widely applied in the field of engineering construction and it is proved effective. But if geologic conditions of the construction are complex and the model is large, the conventional modeling method is difficult, time-consuming and inefficient. For that purpose, we decide to use a new modeling method, which is based on the RHINO-KUBRIX and it is intuitive and fast. For example of unfavorable geologic sections of Lirang tunnel in Liangzhong highway, use this method to build 3D numerical tunnel models under a variety of stratums and calculate the ground stress of models, then get the tunnel local stress of surrounding rocks and use FLAC3D to simulate the excavation of tunnel, and through the comparison of $\mathrm{Z}$ direction deformation of tunnel lining and monitoring data, analyze the interaction of lining and surrounding rocks in tunnel excavation, then verify the feasibility and the validity of this modeling method, so the research results can provide reference for the numerical modeling in complex geological conditions.
\end{abstract}

\section{Preface}

FLAC3D (Fast Lagrangian Analysis of Continua) is based on the continuum of fast Lagrangian analysis method, which has an advantage about the calculation of rock mass stress and deformation calculation, and it has gradually become an ideal 3D numerical calculation tool for the personnel engaged in scientific research and engineering, which is widely applied in transportation, mining and other engineering fields ${ }^{[1]}$. In the field of transportation, FLAC3D can be used for the analysis in the process of tunnel excavation about the interaction between surrounding rocks and supporting structure as well as the deformation of the supporting structure, and to optimize the supporting parameters through the theoretical calculation so as to promote the tunnel construction. Yet since FLAC3D is comparatively easy to model the simple geological models, there are many technical difficulties in building the large complex geological body, and the model is low accurate, the process of modeling is also time-consuming.

Therefore, it has become a new exploration direction in the field of geological engineering about how to combine the efficient 3D modeling with the powerful numerical analysis of FLAC3D. Many scholars have done much fruitful work ${ }^{[2-9]}$, for example, Liao Qiulin ${ }^{[2]}$ writes a FLAC3D-ANSYS interface program using the Visual Basic, making FLAC3D modeling intuitive, fast and automatic. $\mathrm{Hu} \mathrm{Bin}{ }^{[3]}$ programs the FLAC3D pre-processing program via the Fortran, making the modeling of complex FLAC3D calculating model faster and more accurate. Wang Shuren ${ }^{[4]}$ puts forward the method which uses the MIDAS/GTS for modeling the 3D geometric body and meshing, then based on the data relationship of variance analysis about the cells and the nodes of these two kinds of software, use MATLAB to program the MIDAS/GTS-FLAC3D interface program, making the data conversion which imports the modeling information of nodes and cells to the FLAC3D in MIDAS/GTS environment. Lin Hang ${ }^{[5]}$ uses the Fortran to program the SURPAC-FLAC3D interface program, making modeling of FLAC3D high-speed and expanding the application spaces of FLAC3D and SURPAC. Gao Shengxiang ${ }^{[6]}$ completes the modeling of complex geological body by writing MATLAB interface program to generate the FISH Script. 
The researches above have improved the efficiency of the construction of FLAC3D numerical model to a great extent, but these methods, which are all converting models of other numerical software through interface program into FLAC3D models, are limited by the modeling ability of other numerical software and the programming capacity of application personnel, making it difficult to achieve the complex modeling of geographic models which contain multiple stratums and complicated shapes. Therefore, based on the 3D graphic software RHINO and interface program KUBRIX, this article builds a 3D numerical model of tunnel with complicated geographic conditions and multiple stratums, getting the local stratums of initial ground stress through the calculation of the original rock stress of the whole model, analyzing the deformation of the tunnel under excavation according to the given ground stress, to verify the feasibility of RHINO-KUBRIX modeling method.

\section{Instruction of RHINO-KUBRIX}

Rhinoceros (referred to as RHINO) is a piece of drawing software based on NURBS(Non-Uniform Rational B-Splines), and it is widely used in 3D animation, industrial manufacturing, mechanical design and other fields. The powerful capacity of surface building and high-quality model structure generation make it convenient and efficient to create very precise 3D model surface although the software is small and has low request of computer hardware.

KUBRIX is a piece of dedicated software which is designed by ITASCA Company for its FLAC3D, PFC3D and 3DEC analysis software to build high-quality grid models. It is also an automatic mesh generator which can integrate and cooperate with the entity modeling software including RHINO, as well as control the size and accuracy of grid units intelligently and efficiently.

The principle that RHINO and KUBRIX comply with when they work together is: first, import the triangle grids generated in RHINO (STL file), then set the grid parameters that the FLAC3D calculation models need in KUBRIX, including max edge length of grid, gird level, node offset that the grid generated compares with the imported, as well as parameters set of cutting angle, etc. After parameters set, KUBRIX will read the number information of triangle mesh surface and nodes automatically, check for the self-intersection of triangle mesh surface after model is given the section property as well as repair it, and mesh the models again according to the parameters set before, until FLAC3D grid files (flac3d) and detailed text files of numerical models are generated successfully.

\section{Engineering application}

\section{Project profile}

In the background of Lirang highway tunnel in the Liangping county of Chongqing, this paper executes the 3D modeling about the complex geologic sections of Lirang tunnel. The length of left line of tunnel is $5517.6 \mathrm{~m}$, the right is $5527.0 \mathrm{~m}$. The tunnel is characterized as a long and big-span tunnel with a variety of unfavorable geologic conditions such as gas, gypsum rocks, the mined-out areas, coal seams, karst, etc. The existence of a variety of unfavorable geologic conditions brought engineering construction serious difficulties, posing serious threat to the safety of construction personnel at the same time. In order to optimize the construction, we use the numerical software to exceed the theoretic numerical calculation about the excavation project, supporting structure and the deformation as well as the interaction between surrounding rocks and supporting structure in the construction of tunnel. The first step of optimization for all kinds of calculation and projects is to build the 3D numerical model of excavation tunnel. The 3D model of Lirang tunnel this paper builds is in the range of mileage pile number ZK13 + 550 ZK13 + 550 in left tunnel hole, and the length of this model is $1900 \mathrm{~m}$ while the width is $200 \mathrm{~m}$.

\section{Numerical modeling of Lirang tunnel}

From bottom to top, RHINO builds the entity model by dots, lines, faces and bodies in order. Powerful entity segmentation, Boolean operation can realize complex computation between entities such as add, subtract, combination, etc. Open the engineering design CAD drawing in RHINO, obtain the physical model by calculation, and mesh the entities, so we can control cell size, shape and 
refinement level of grids. Finally, generate the RHINO mesh files (STL) and import them into KUBRIX so as to regenerate the FLAC3D grid files. The detailed steps are shown below:

(1) According to the design project, use RHINO to build the section of tunnel that goes through the unfavorable geologic sections, and preprocess the part that has nothing to do with the calculation object. As shown in the Fig. 1:

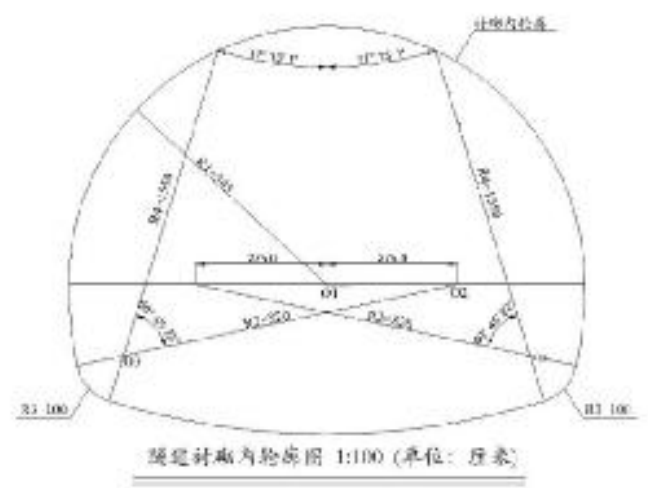

Fig. 1 Tunnel cross section profile

(2) According to the tunnel vertical section profile in the design project, tunnel profile geological map in RHINO was built in terms of certain proportion. As shown in Fig. 2:

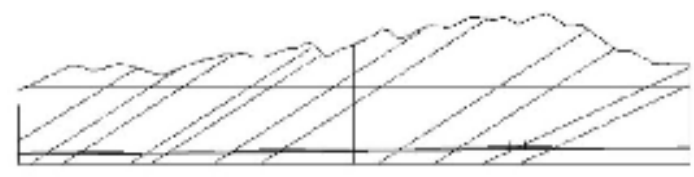

Fig. 2 Tunnel vertical section profile

(3) In RHINO, place the Tunnel cross section profile (Fig. 1) to the corresponding position in Tunnel vertical section profile (Fig. 2), and stretch along the tunnel longitudinal whose mode is entity extrusion. As shown in Fig. 3:

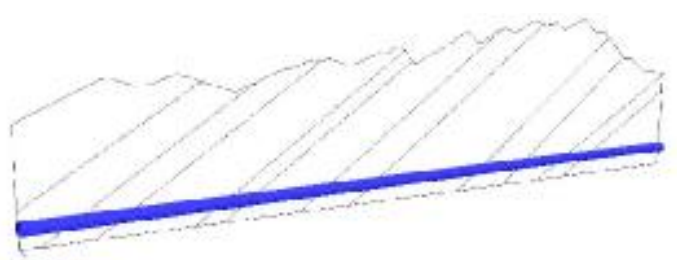

Fig. 3 Tunnel stereogram across pitch

(4) Also in RHINO, build the model "GROUP" of different stratums through "extrusion entity", "Boolean operation", "split", "trim" and so on, and indicate them with different colors. Particular way is to extrude the entire model boundary and the various stratums out $100 \mathrm{~m}$ on each side, whose way of extrusion is entity extrusion, to make the width of tunnel entities $200 \mathrm{~m}$ and indicate the numerical models with different "GROUP". As shown in Fig. 4: 


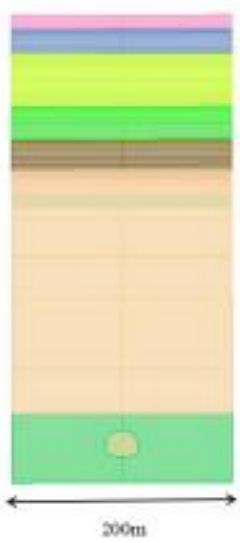

(a) Cross section profile of numerical model

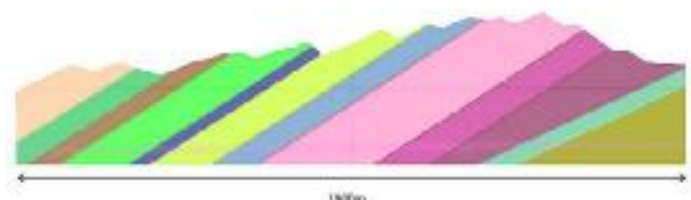

(b) Vertical section profile of numerical model

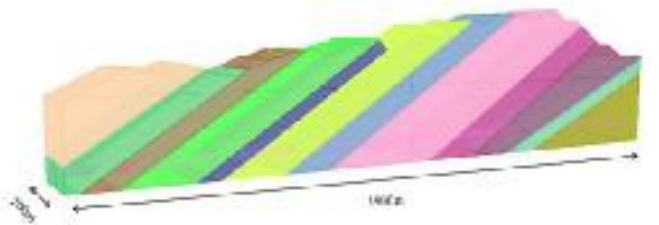

(c) 3D figure of numerical model

Fig. 4 Tunnel model

(5) In RHINO, combine the "GROUP" represented the different stratums in a way of entity combination into an entire entity model. As shown in Fig. 5:

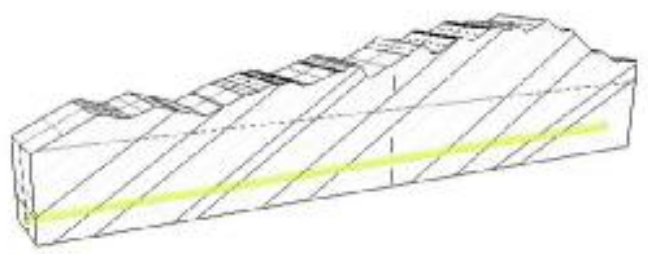

Fig. 5 Tunnel model of unfavorable geological conditions

(6) In RHINO, combine each non-manifold surface to generate tunnel entities, then convert them into grid models, and next, triangulate the grid models, output the triangular grid models of ASCII format (STL file). As shown in Fig. 6:

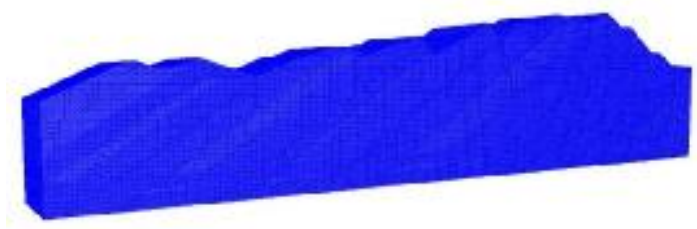

Fig. 6 Triangulated STL file exported by RHINO 
(7) After related parameters set in KUBRIX, divide the mesh surface of the output grid file (STL file) again, and ultimately generate the FLAC3D grid files of tetrahedron and hexahedron (flac3d file). As shown in Fig. 7, 8:

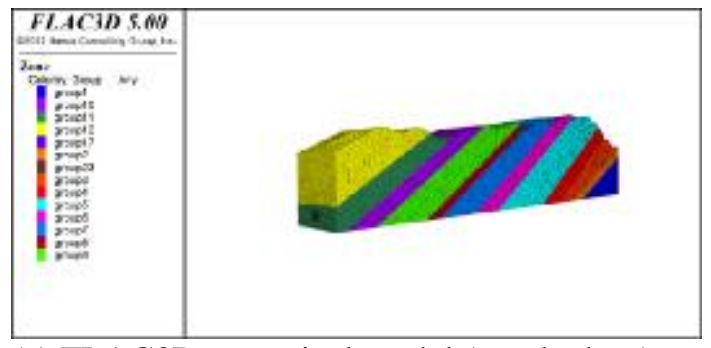

(a) FLAC3D numerical model (tetrahedron)

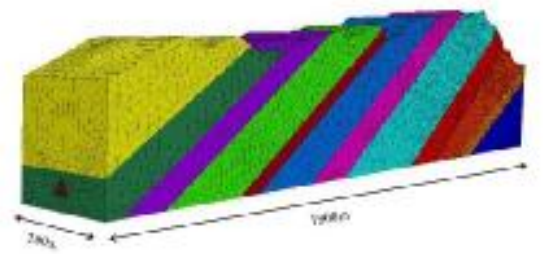

(b) FLAC3D numerical model (tetrahedron)

Fig. 7 FLAC3D tetrahedral grid model

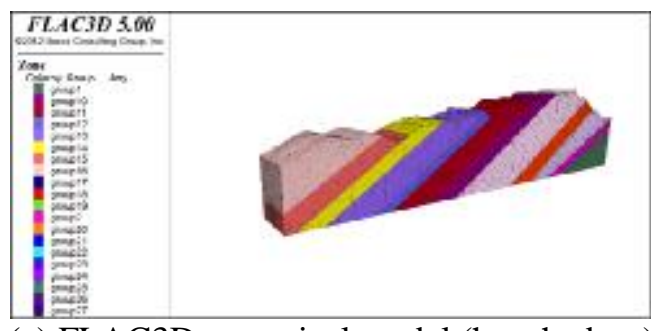

(a) FLAC3D numerical model (hexahedron)

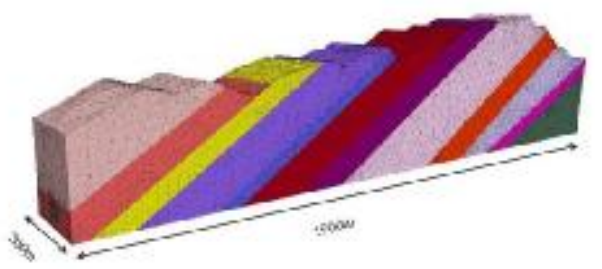

(b) FLAC3D numerical model (hexahedron)

Fig. 8 FLAC3D hexahedral grid model

Node matching validation in FLAC3D shows that the connection between each node is good. The entire modeling process takes 3 hours, but if we use conventional method, it will take at least 1 week and the precision is low. Compared with conventional modeling method in modeling efficiency and accuracy, RHINO-KUBRIX method has more advantages, with much time saved in modeling process, more beautiful output model, higher quality of model grid and properly division of different stratums, and the numerical model also basically meets the requirements.

\section{Calculation of initial ground stress in Lirang tunnel}

According to geological survey data gotten in Lirang tunnel, we can obtain the rock mechanics parameters of different stratums in numerical model, and use Mohr-Coulomb strength criterion for 
elastic solution of tetrahedral grid model and hexahedral grid model respectively in FLAC3D program.

Initial ground stress contour of tetrahedral grid model is shown in Fig. 9:

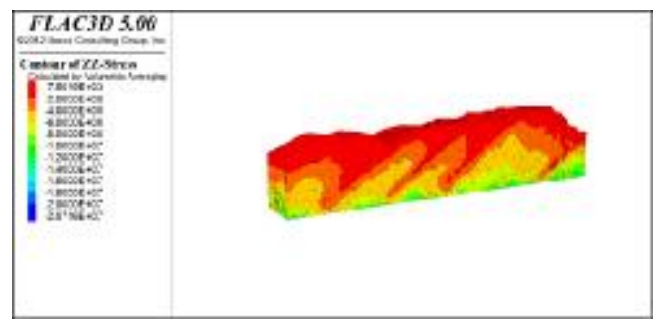

Fig. 9 Initial ground stress field of tetrahedral grid model

Initial ground stress contour of hexahedral grid model is shown in Fig. 10:

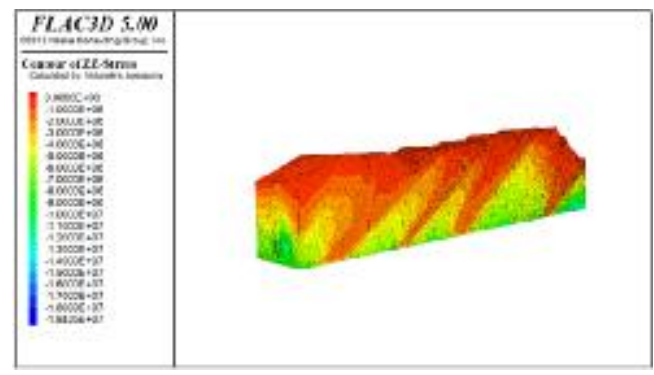

Fig. 10 Initial ground stress field of hexahedral grid model

From Fig. 9 and Fig. 10, we can know the max ground stress in tetrahedral grid model is 20.7Mpa, while it is $18.4 \mathrm{Mpa}$ in hexahedral grid model. According to the construction data in Lirang tunnel, we can know that the ground stress field obtained from tetrahedral grid model is close to the one from engineering practice. The result of larger error in calculation for this kind of hexahedral grid would be the process which divides tetrahedral grid into several hexahedral grids and makes mesh more tedious, as well as the disorder of connection between adjacent grid nodes. Hence, tetrahedral grid is better than hexahedral one no matter in precision of model or in accuracy of calculation results.

The ground stress obtained from calculation will be used in analysis of the $\mathrm{Z}$ direction deformation of lining structure in the full section excavation in the range of mileage pile number ZK13 + 550 ZK13 + 550 in left tunnel hole.

\section{Analysis of lining structure deformation}

As shown in Fig. 11, the dimension of numerical calculation model is $40 \mathrm{~m} \times 40 \mathrm{~m} \times 40 \mathrm{~m}$, and the cross section of grid model is also shown below:

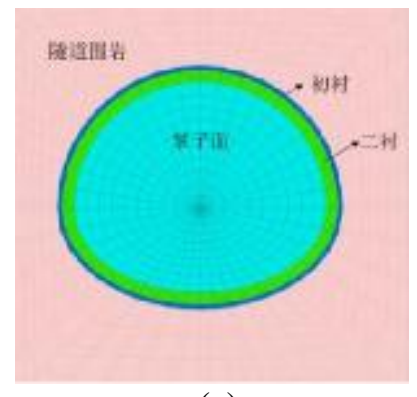

(a) 


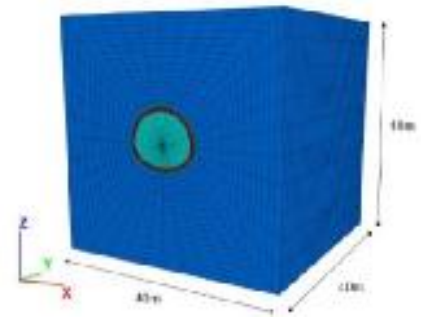

(b)

Fig. 11 Fine surrounding rock mass of tunnel grid model

The whole section excavation method is used to the tunnel model in Fig. 11, while the footage of excavation is $3 \mathrm{~m}$, then do the shotcrete support (primary support) immediately after the excavation, and do the second support $6 \mathrm{~m}$ far from the tunnel face. The diagram of tunnel excavation is shown in Fig. 12:

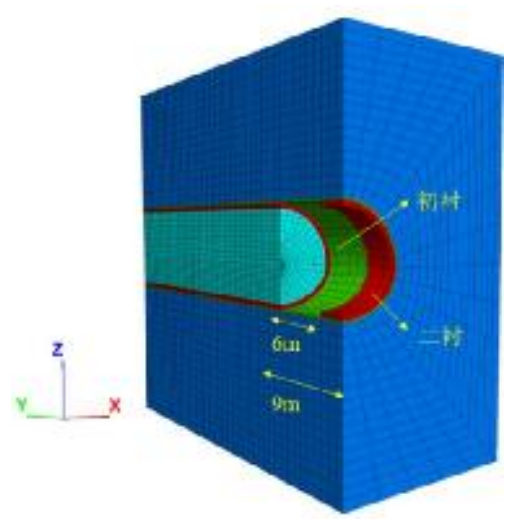

Fig. 12 Diagram of tunnel excavation

Apply the initial ground stress of tunnel surrounding rocks gotten from the last section (as shown in Fig. 10) into the numerical model (shown in Fig. 12), and study the effect that excavation has on the deformation and stress of lining structure. Calculate in 4 excavation cycles ( 2 of modeling and 2 of lining), and analyze the structure deformation of primary lining and second lining.

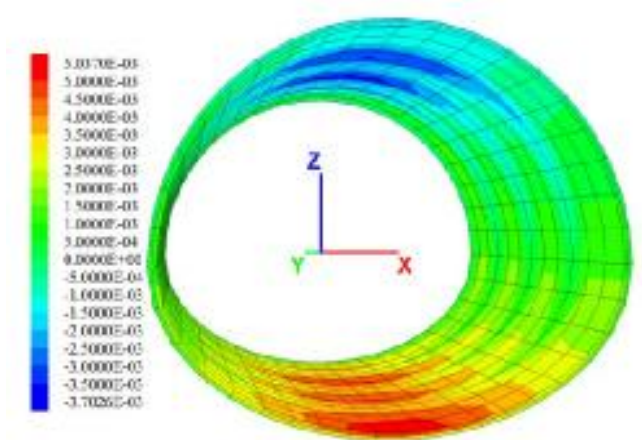

(a) Z direction displacement of primary lining structure 


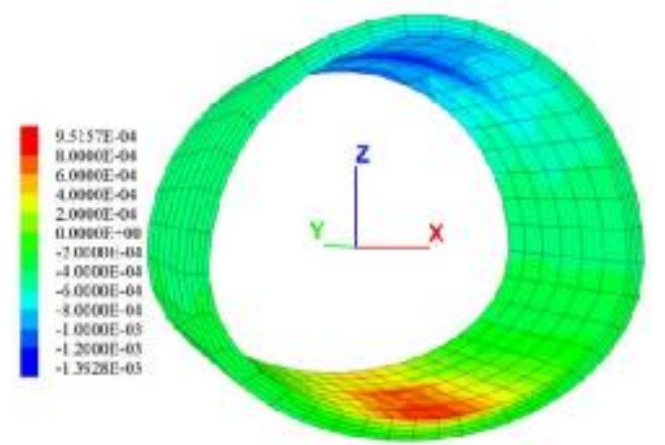

(b) Z direction displacement of second lining structure

Fig. $13 \mathrm{Z}$ direction displacement contour of lining structure

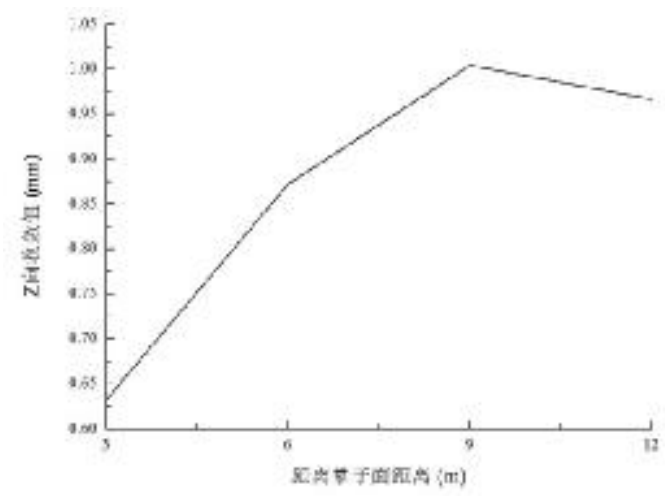

Fig. $14 \mathrm{Z}$ direction converge curve

After 4 cycles simulated excavation of Lirang tunnel, we obtain the $\mathrm{Z}$ direction displacement contour of primary lining and second lining by calculation, as shown in Fig. 13, and the Z direction displacement of lining deformation is positive. Fig. 14 is the $\mathrm{Z}$ direction converge curve of monitoring points, two monitoring points are respectively located at the tunnel vault and the corresponding arch bottom. Combined with Fig. 13 and Fig. 14, it can be seen that tunnel excavation releases the rock stress, with convergence deformation occurred in tunnel surrounding rocks, vault sinking and arch bottom uplifting, so it is supposed to timely shotcrete to close the bare rocks in order to avoid the large deformation of surrounding rocks, and at this time the primary lining structure has also happened a bit of deformation due to the compression of rocks, as shown in Fig. 13(a), reflecting the interaction and together-bearing between surrounding rocks and supports. As the tunnel is being excavated, deformation of surrounding rocks and supports gradually changes, but still on the rise, as shown in Fig. 14. When monitoring points are $9 \mathrm{~m}$ from tunnel face, do the second lining, at this time converge curve doesn't change a lot, and the deformation doesn't increase, and the second lining also reaches the surrounding rocks. The extrusion between rocks and primary supports produces deformation, but it is very small, as shown in Fig. 13(b). From Fig. 13 and 14, we can see the deformation of supporting structure in tunnel excavation, the interacted deformation and together-bearing can also be illustrated.

\section{Conclusion}

This paper adopts a new numerical modeling method, building FLAC3D numerical model of Lirang tunnel in complex geologic sections based on RHINO-KUBRIX. Then obtain the local initial ground stress field of numerical model calculated by FLAC3D, as well as the $\mathrm{Z}$ direction deformation of lining structure due to tunnel excavation, and the comparison with monitoring data verifies the feasibility of this method of modeling, at the same time the interacted deformation and together-bearing are also illustrated. 
In the aspect of modeling, because RHINO has a powerful drawing function, with operation similar to the CAD, and learning it is relatively easy, so engineers can use RHINO graphic processing function to build 3D entity models, view and modify them as long as the geometry data points are obtained, then with the help of KUBRIX's high-quality mesh generation and the perfect cohesion of both and FLAC3D, we can finish the early model generation phase in numerical calculation. Apply this method to the numerical model building in complex geologic section of Lirang tunnel in Liangzhong highway, and verify the feasibility and effectiveness of this method.

This method is easy to operate, with more accurate output model, and the entire processes are all visual operation and straightforward, greatly reducing the time and energy needed for the engineering staff in the conventional modeling, improving the efficiency of the multiple numerical simulation. It not only provides a new effective choice for the engineering staff in modeling, but also is conducive to give full play to the powerful advantages of FLAC3D in solving the problem of geotechnical engineering field.

However, the numerical model still has room to improve further, because in the model the grid density is consistent, making modeling calculation tedious and slow, so that some optimization can be done in the following aspects in future:

(1) Thicken the grid in a certain range of tunnel surrounding rocks, the grid outside the range can be relatively thin providing no influence on model precision;

(2) Due to large model, this numerical model uses the tetrahedral grid, in the future the hexahedral (rectangle) grid model can be tried, so that no matter for the precision or calculation, the result would be more accurate.

\section{References}

[1] Itasca Consulting Group. Fast Lagrangian Analysis of Continua in Three Dimensions [M]. America: Itasca Consulting Group, 1997

[2] Liao Qiulin, Zeng Qianbang, Liu Tong. Automatic model generation of complex geologic body with FLAC3D based on ANSYS platform [J]. Chinese Journal of Rock Mechanics and Engineering, 2005, 24(6): 1010-1013.

[3] Hu Bin, Zhang Zhuoyuan, Huang Runqiu. Pretreatment program development and simulation results testing of FLAC3D [J]. Chinese Journal of Rock Mechanics and Engineering, 2002, 21(9): 1387-1391.

[4] Wang Shuren, Zhang Haiqing. A Coupling Modeling Method with MIDAS/GTS-FLAC 3D and Its Application [J]. Journal of Civil, Architectural \& Environmental Engineering, 2010, 32(1): 12-17.

[5] Lin Hang, Cao Ping, Li Jiangteng. Automatic Generation of FLAC 3D Model Based on SURPAC[J]. Journal of China University of Mining \& Technology, 2008, 37(3): 339-342.

[6] Gao Shengxiang, Ye Rongzhang, Tang Weili. Establishment of complex geological body FLAC3D model by using MATLAB interface program[J]. COAL GEOLOGY \& EXPLORATION, 2009, 37(5): 51-53.

[7] Li Guoqing, Wang Xinqing, Yin Gaimei. Complex FLAC3D three dimensional geological modeling based on moving least squares method[J]. JOURNAL OF GEOMECHANICS, 2013, 19(3):334-342.

[8] Xu Wenjie, Hu Ruilin. Refined modeling of 3D geological body based on reverse engineering and the application of ADINA in the model building of FLAC3D[J]. Journal of Engineering Geology, 2008, 16(5):703-709.

[9] Wang Jilin, Ding Chenjian, Wu Shenglin. 3D GEOLOGICAL MODELING OF COMPLEX LANDFORMS BASED ON FLAC3D[J]. JOURNAL OF GEOMECHANICS, 2008, 14(2). 